\title{
Serous tubal intraepithelial carcinoma: an incidental finding in laparotomy for tubo-ovarian mass in woman aged 35 years
}

\author{
Rinku Kumari*, Mamta Sharma
}

Department of Obstetrics and Gynecology, Government Medical College, Kota, Rajasthan, India

Received: 04 February 2021

Revised: 11 March 2021

Accepted: 12 March 2021

\author{
*Correspondence: \\ Dr. Rinku Kumari, \\ E-mail: ruchiarora940@gmail.com
}

Copyright: (C) the author(s), publisher and licensee Medip Academy. This is an open-access article distributed under the terms of the Creative Commons Attribution Non-Commercial License, which permits unrestricted non-commercial use, distribution, and reproduction in any medium, provided the original work is properly cited.

\begin{abstract}
Serous tubal intraepithelial carcinoma (STIC) is a precursor lesion for high-grade pelvic serous carcinoma. The incidence of STIC is estimated to occur in $0.6 \%$ to $6 \%$ of women who are BRCA positive or have a strong family history of breast or ovarian cancer. We report a rare case of serous tubal Intraepithelial carcinoma in young woman undergoing laparotomy for tubo-ovarian mass who diagnosed STIC on histopathological examination of specimen. Women diagnosed incidentally at surgery for benign condition should be considered for repeat surgical staging. Etiology and management are similar to epithelial ovarian cancer but stage at diagnosis, lymphatic spread and overall survival appear to be different.
\end{abstract}

Keywords: STIC, Surgical staging, Case report

\section{INTRODUCTION}

Primary cancer of fallopian tube is rare malignancy of female genital tract. It accounts for between $0.1-1.8 \%$ of all gynaecological cancers diagnosed. ${ }^{1,2}$ Commonest age 50-60 year (mean age-55 years). ${ }^{3}$ Bilateral disease is uncommon and represents fewer than $25 \%$ cases. ${ }^{4}$ Serous tubal intraepithelial carcinoma (STIC) is a rare pathologic finding at the time of benign gynecologic surgery. It arises in the distal fimbriated end of the fallopian tube and likely represents a precursor lesion to high-grade pelvic serous carcinoma.

\section{CASE REPORT}

In January 2020, a 35-year-old P3L3 presented with complaining of acute pain abdomen since 2-3 days and heaviness in lower abdomen since 1 month. Her obs history was 3 full term L.S.C.S. with abdominal sterilization 7 years back. No significant past medical and family history. On per abdomen examination- transverse scar present and Abdominopelvic lump around 18 weeks size which is mobile, cystic and tenderness on deep palpation. On bimanual examination uterus size not made out due to obesity. Her blood investigation was normal and her CA-125 was normal. Her pap smear report shows fungal organisms consistent with candida species. USG report was large cystic mass with internal echoes seen in right lower abdomen. CECT abdomen \& Pelvis revealed large thick-walled cystic lesion in right adnexa likely benign cystic lesion of right ovary. Laparotomy planned and intraoperative findings were right tubo-ovarian mass (size around $15 \times 20 \mathrm{~cm}$ ), tube entirely stretched over ovarian mass, Mass was smooth, cystic, mobile with torsion and no ascitic fluid in peritoneal cavity. Contralateral ovary and fallopian tube was normal. Hence Right salphingo-oopherectomy was planned and done. Same sent for HPE. Her histopathological examination report revealed mucinous cystadenoma of ovary and serous tubal intraepithelial carcinoma. Advised her IHC p53, p16, ki67. 


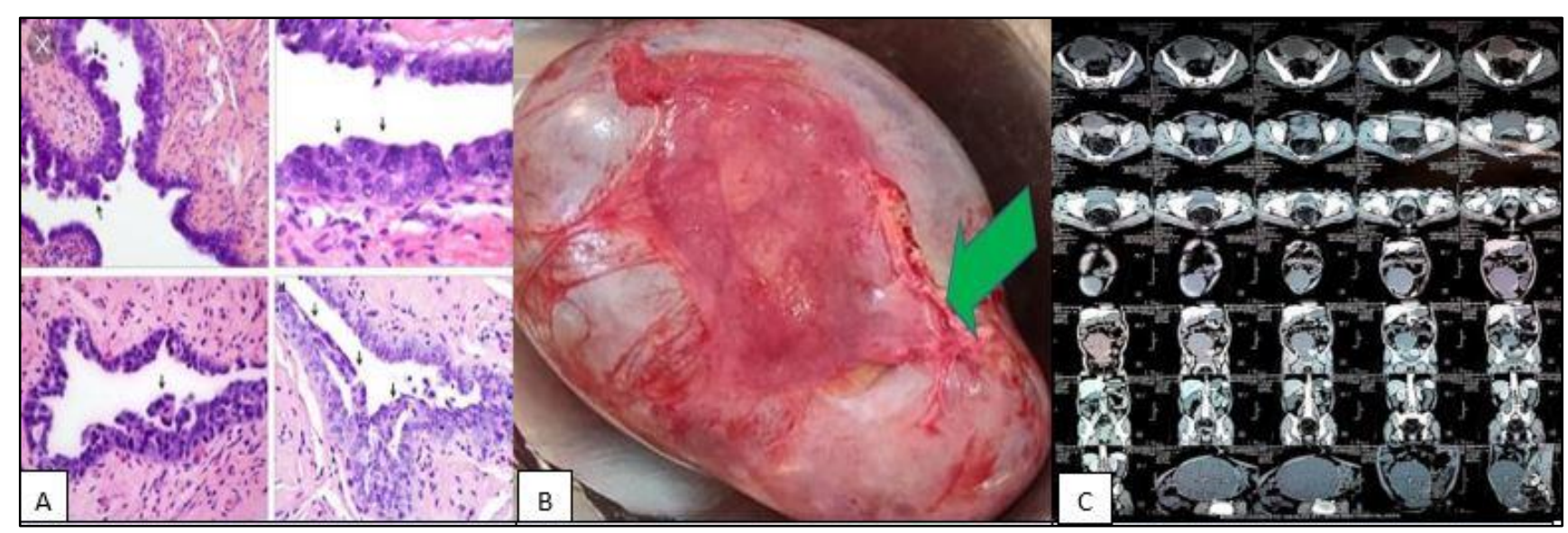

Figure 1: (A) Microscopic view of STIC, (B) gross specimen, (C) radiological finding in CECT.

\section{DISCUSSION}

Primary fallopian tube cancers- $>90 \%$ cases are papillary serous adenocarcinoma (Histology dominance-serous type). ${ }^{1}$

Fallopian tube cancers mostly secondary to other primary malignancy of ovary, endometrium, GIT, breast and peritoneal carcinomatosis. ${ }^{5}$

Preoperative diagnosis of primary fallopian tube malignancy is very difficult, especially in earlier stages (Incidence-0.3\%). ${ }^{6}$ Women who are BRCA positive or have a strong family history of breast or ovarian cancerincidence increases to 0.6 to $6 \% .^{7-9}$ If there are symptoms, abnormal vaginal bleeding or profuse watery vaginal discharge are the commonest. These may be associated with vague lower abdominal pain, abdominal distension and pressure. Fewer than $10 \%$ of women present with the classic description of hydrops tubae profluens: a palpable pelvic mass that resolves during examination and is associated with a watery vaginal discharge.14 Rarely, it can manifest as an acute abdominal emergency with pelvic pain. Clinical examination will sometimes reveal an adnexal mass. ${ }^{10}$

The management of fallopian tube cancer same as that for ovarian cancer. ${ }^{11}$

The modality of treatment is Surgical-washings of peritoneal cavity followed by TAH with BSO along with sampling of pelvic and paraaortic lymph nodes, infra colic omentectomy and appendicectomy (similar treatment was advised to the patient). ${ }^{10}$

Follow-up: annual review of systems, pelvic examination, tumor markers or imaging.

Over all five year survival rate of patients with epithelial tubal cancer is about $33-40 \%$ with reported symptom free survival rate of up to $21 \% .^{11}$

\section{CONCLUSION}

Women diagnosed incidentally at surgery for a benign condition should be considered for repeat surgical staging. Etiology and management are similar to epithelial ovarian cancer but stage at diagnosis, lymphatic spread and overall survival appear to be different.

Funding: No funding sources Conflict of interest: None declared

Ethical approval: Not required

\section{REFERENCES}

1. Benedet JL, Bender H, Jones H 3rd, Ngan HY, Pecorelli S. FIGO staging classifications and clinical practice guidelines in the management of gynecological cancers. FIGO Committee on Gynecologic Oncology. Int J Gynaecol Obstet. 2000;70:209-62.

2. Woolas R, Jacobs I, Davies AP, Leake J, Brown C, Grudzinskas JG, et al. What is the true incidence of primary fallopian tube carcinoma. Int $\mathbf{J}$ Gynecol Cancer. 1994;4:384-8.

3. FIGO Report. Carcinoma of the fallopian tube: patients treated in 1990-1992 - distribution by age groups. J Epidemiol Biostat. 1998;3:93.

4. Yuen JH, Wong CG, Lam CH. Preoperative sonographic diagnosis of primary fallopian tube carcinoma. J Ultrasound Med. 2002;21:1171-3.

5. Inal MM, Hanhan M, Pilanci B, TinarS. Fallopian tube malignancies: experience of Social Security Agency Agean Maternity Hospital. Int J Gynecol Cancer. 2004;14:595-9.

6. Eddy GL, Copeland LJ, Gershenson DM, Atkinson EN, Wharton JT, Rutledge FN. Fallopian tube carcinoma. Obstet Gynecol. 1984;64:546-52.

7. Finch A, Shaw P, Rosen B, Murphy J, Narod SA, Colgan TJ. Clinical and pathologic findings of prophylactic salpingo-oophorectomies in 159 BRCA1 and BRCA2 carriers. Gynecologic Oncology. 2006;100(1):58-64. 
8. Wethington SL, Park KJ, Soslow RA. Clinical outcome of isolated Serous tubal intraepithelial carcinomas (STIC). International Journal of Gynecological Cancer. 2013;23(9):1603-11.

9. Manchanda R, Abdelraheim A, Johnson M. Outcome of risk-reducing salpingo-oophorectomy in BRCA carriers and women of unknown mutation status," BJOG: An International Journal of Obstetrics and Gynaecology. 2011;118(7):814-24.

10. Romagosa C, Torne A, Iglesias X, Cardesa A, Ordi J. Carcinoma of the fallopian tube presenting as acute pelvic inflammatory disease. Gynecol Oncol. 2003;89:181-4.

11. Goswami PK, Kerr-Wilson R, McCarthy K. Cancer of the fallopian tube. The Obstetrician \& Gynaecologist. 2006;8:147-52.

Cite this article as: Kumari R, Sharma M. Serous tubal intraepithelial carcinoma: an incidental finding in laparotomy for tubo-ovarian mass in woman aged 35 years. Int J Reprod Contracept Obstet Gynecol 2021;10:1698-700. 